\title{
Diabetes Mellitus: Metabolic Effects and Oxidative Stress ${ }^{1}$
}

\author{
I. B. Zavodnik ${ }^{a, b}$, I. K. Dremza ${ }^{a}$, E. A. Lapshina ${ }^{a}$, and V. T. Cheshchevik ${ }^{a}$ \\ ${ }^{a}$ Institute of Pharmacology and Biochemistry, National Academy of Sciences of Belarus, BLK-50, Grodno, 230017 Belarus; \\ fax: +375 152 434121; e-mail: zavodnik_il@mail.ru \\ ${ }^{b}$ Department of Biochemistry, Yanka Kupala State University, ul. Oszeshko, 22, Grodno, \\ 230022 Belarus@ \\ Received August 8, 2010
}

\begin{abstract}
Diabetes mellitus is a complex polygenic pathology, which is characterized by numerous metabolic disorders. Progressive hyperglycemia developing during this disease causes clinically significant tissue damage and is considered as a main risk factor of micro- and macrovascular complications leading to retinopathy, nephropathy, and neuropathy. Hyperglycemia-depended oxidative stress and impairments in nitric oxide bioavailability play an essential role in the pathogenesis of diabetes and its complications. Homeostasis of glucose maintained by metabolic effects of insulin includes an increase of glucose uptake by skeletal muscles and suppression of glucose production by the liver. M. Brownlee (2005) put forward a hypothesis assuming that oxidative stress is the main mechanism of diabetic tissue damages. According to this hypothesis, mitochondrial dysfunction and superoxide anion radical hyperproduction by mitochondria is the principal mechanism of activation of four pathways of hyperglycemia-induced impairments under diabetes. Two cell signaling cascades regulate the glucose homeostasis: insulin-mediated glucose uptake (IMGU) in skeletal muscles, liver, and heart and glucose-stimulated insulin secretion (GSIS) in pancreatic $\beta$-cells. In addition to nonspecific irreversible oxidative damage of DNA, protein and lipid molecules reactive oxygen and nitrogen species induce cell and tissue damage, activating a number of cell stress-sensitive signaling cascades. Stress-dependent serine phosphorylation of insulin receptor substrate (IRS) proteins decreases its capacity for tyrosine phosphorylation and may accelerate degradation of IRS. This process underlies the molecular mechanism of oxidative stress-induced insulin resistance.
\end{abstract}

Keywords: diabetes mellitus, insulin, cellular signalization, oxidative stress, mitochondria.

DOI: $10.1134 /$ S1990747811020097

\section{PATHOBIOLOGY OF HYPERGLYCEMIA. THE ROLE OF OXIDATIVE STRESS}

Insulin-dependent and insulin-independent diabetes mellitus stands among the most prevalent pathologies of modern society. A menacing growth of the number of diabetes mellitus cases and the price paid for the maintenance of public health make imperative the task to understand pathophysiological mechanisms of this pathology and develop approaches allowing its prevention.

Diabetes mellitus is a complex polygenic pathology, which is characterized by multiple methabolic disorders. Epidemiological studies confirm that the cause of clinically recorded tissue damage during diabetes mellitus is progressive hyperglycemia [1], the most important risk factor of subsequent micro- and macrovascular complications, leading to diabetic retinopathy, nephropathy, and neuropathy. Oxidative stress and disorders in nitrogen oxide availability, accompanying hyperglycemia, play an important role in the pathogenesis of both diabetes mellitus and its complications $[2,3]$. The mechanisms leading to gen-

\footnotetext{
${ }^{1}$ The article was translated by the authors.
}

eration of reactive oxygen and nitrogen species and to oxidative stress, include metabolic stress caused by disorders in cell bioenergetics, glucose auto-oxidation $[3,4]$, synthesis of inflammatory mediators, as well as non-enzymatic glycosylation of proteins and lipids. It should be emphasized that tissue damage during diabetes mellitus is determined by processes taking place at the molecular and cellular levels. Thus, the development of diabetes mellitus type 1 and its symptoms are linked to dysfunction of pancreatic $\beta$-cells, while in the case of diabetes mellitus type 2 , the sensitivity of receptors of target cells to insulin (insulin resistance) and glucose uptake by tissues are disturbed.

It should be noted that the most common chronical non-infectious diseases, such as cancer, diabetes mellitus, cardiovascular and neurodegenerative diseases, are age-related, polygenic, polyfunctional pathologies, often having a considerable non-enzymatic, non-metabolic, and chemical components [5].

Metabolic effects of hyperglycemia. Glucose homeostasis maintained by insulin includes an increase in glucose uptake by skeletal muscles and suppression of glucose production in liver. M. Brownlee put forward an idea about the role of oxidative 
stress in the development of diabetes mellitus [1]; according to this hypothesis, mitochondrial dysfunction and overproduction of superoxide radicals by mitochondria is the main mechanism of activation of hyperglycemia-dependent metabolic pathways of tissue damage during diabetes mellitus. Chronical tissue lesions, developing during diabetes mellitus and similar diseases, include both oxidative modification of proteins, DNA and lipids by reactive oxygen and nitrogen species and chemical modification of proteins (and not only proteins) by the products of sugar and lipid oxidation. In these processes, excessive end products of glucation/glucoxidation and lipoxidation (AGE/ALE) are formed [6]. The increase of glucose level induces metabolic and physiological signals having the same mechanisms in pancreas and peripheral tissues, yet producing different results because of functional specialization of tissues [7].

Transport of glucose into a cell by tissue-specific transporters is not a rate-limiting process. The next step of glucose metabolism is its phosphorylation by tissue-specific glucokinases. In $\beta$-cells, glucokinase (or hexokinase IV) is activated after binding to poreforming protein of the outer mitochondrial membrane (in the site of contact of the inner and outer mitochondrial membranes). Similar mechanism of hexokinase II activation exists in skeletal muscle and adipose tissue. Activation of hexokinase depends on the pore structure, which is voltage-dependent and is regulated by electric potential of the inner mitochondrial membrane. Defects of respiratory chain leading to depolarization of mitochondrial membrane can be linked to abnormal activities of enzymes, phosphorylating glucose. Interaction of hexokinase with the contact site of the mitochondrial membranes and its activation lead to an increase of ADP and glucose-6-phosphate (G-6P) levels. ADP goes directly into mitochondria, stimulating oxidative phosphorylation. G-6-P is an extremely important intermediate of energy metabolism, standing on key position of reaction switching between glycolysis, glycogen synthesis and the pentose phosphate pathway. Glycolysis is linked with reactions of mitochondrial oxidative phosphorylation, which accelerates during an increase of the blood glucose level, in three points: transport of NADH to complex I of respiratory chain through malate/aspartate transporter, transport of $\mathrm{FADH}_{2}$ to complex II through the glycerophosphate/ hydroxyacetonephosphate cycle, and transport through hexo(gluco)kinases, donating ADP to complex V (ATP-synthase). In cerebral and muscle cells phosphocreatine transporter can also be involved in glucose-induced signaling cascade, resulting in insulin secretion by $\beta$ ]-cells.

Interaction between plasma membrane-associated and mitochondrial creatine kinases provides a local increase of ATP concentration near ATP-dependent ion channels. This leads to an increase of cytosolic and even intramitochondrial calcium concentration and, as a result, to insulin secretion. Thus, in $\beta$-cells glu- cose stimulates phosphocreatine synthesis in mitochondria by membrane-bound glucokinase. A similar sequence of signals is used in muscle tissues in reverse direction, when during physical activity creatine level increases due to ATP exchange, which in turn stimulates synthesis of ATP in mitochondria and phosphorylation of glucose by hexokinase. Moreover, mutual influence of cytosol and mitochondria promotes activation of glycogen synthesis by glucose. The activity of mitochondria-bound hexokinase provides G-6-P synthesis and stimulates UTP production with participation of mitochondrial nucleoside diphosphate kinase [7].

Pathophysiologically, at least two forms of diabetes mellitus are recognized, which differ in energy metabolism. The first form is determined by point mutation of glucokinase gene, while the second form is represented by several forms of mitochondrial diabetes, which has its origin in mutations of mitochondrial DNA (mtDNA), encoding several subunits of mitochondrial respiratory chain. mtDNA is very sensitive for damage and accumulates mutations during ageing [7]. The origin of diabetes mellitus type 2 (and also the metabolic syndrome) is a resistance of tissues to insulin, i.e., a loss of the ability of hormone to suppress glucose efflux from liver cells and provide peripheral glucose disposal, as well as lack of the ability to mask relative $\beta$-cells dysfunction.

During diabetic hyperglycemia, four main metabolic processes playing important roles in tissue damage are activated [1].

1) The polyol metabolic pathway; hyperglycemia leads to increase of the rate of enzymatic conversion of glucose to polyatomic alcohol (polyol) sorbitol (aldose reductase, the first and rate-limiting enzyme of the polyol metabolic pathway, catalyses glucose reduction to sorbitol). Sorbitol is metabolized to fructose by sorbitol dehydrogenase, increasing $\mathrm{NADH} / \mathrm{NAD}^{+}$ratio. The activity of this alternative metabolic pathway significantly increases at high concentrations of glucose, the substrate of hexokinase responsible for conversion of glucose to G-6-P; meanwhile, the availability of glucose for aldose reductase increases dramatically [8]. Under these conditions, sorbitol, which is slowly metabolized and slowly diffuses through cell membranes, is accumulated in the cell. This leads to a misbalance in cell homeostasis and to the development of long-term complications of diabetes mellitus [9, 10].

2) Generation of stable advanced glycation end products (AGE), their autooxidation and interaction with cellular receptors. Reducing sugars (glucose, G6-P, fructose) with aldehyde groups interact with free amino groups of proteins, forming reversible Schiff bases. Slow Amadori rearrangement of Schiff bases leads to the AGE formation. As a result, protein structures and functions are modified, causing stable cell damage. Moreover, cells have specific receptors to AGE [11]. 
3) Activation of protein kinase $\mathrm{C}$ (PKC) isoforms. The process of glucose metabolism can be accompanied by increased rate of fatty acid synthesis and generation of lipid derivatives acting as signaling molecules (for example, diacylglycerol (DAG)) and potential activators of PKC [12].

4) Increase of glucose flow through the hexosamine pathway. A number of toxic effects of glucose are related to biosynthesis of hexosamines, products of one of the minor pathways of glucose metabolism [13], which is activated during hyperglycemia. Normally, $2-5 \%$ of glucose received by cell is metabolised through the hexosamine pathway. The hexosamine pathway starts from glutamine: fructose-6-phosphate amine transferase-catalysed conversion of fructose-6phosphate to glucosamine-6-phosphate [14]. The end product of this metabolic pathway, UDP-N-acetylglucosamine, takes part in glycosylation of a number of intracellular proteins, particularly transcription factors, so it may have an effect on transcription of a number of genes [15].

Reactive oxygen species (ROS) generated during hyperglycemia can suppress activity of glyceraldehyde-3-phosphate dehydrogenase, leading to accumulation of intermediates of the glycolytic pathway. One of such intermediates, glyceraldehyde-3-phosphate (along with fructose-6-phosphate) significantly increases the AGE generation. The diacylglycerol/PKC-dependent signaling pathway stimulates the hexosamine pathway [16]. In these cases, hyperproduction of ROS by electron transport chain of mitochondria stimulated by increased level of glucose utilized in the Krebs cycle (and by increase of concentration of electron donors NADH and $\mathrm{FADH}_{2}$ ) can be considered as a general mechanism integrating independent biochemical processes numbered above [1].

Diabetes mellitus and stress-dependent signaling cascades. Hyperglycemia activates not only well-characterized biochemical processes, such as formation of stable glycosylation/glycation products and their interaction with corresponding receptors [17], the polyol pathway [18], but also stimulates a series of stress-dependent signaling cascades, playing a role in cell damaging and leading to development of longterm complications of diabetes mellitus [19]. One of the well-studied signaling cascades is that associated with transcription factor NF- $\mathrm{B}$ [20]- $\mathrm{a}$ target of ROS, hyperglycemia and oxidative stress. NF- $\kappa \mathrm{B}$ regulates expression of a variety of genes related to diabetic complications, atherosclerosis, and promoting inflammatory response and apoptosis. Activation of $\mathrm{NF}-\kappa \mathrm{B}$ includes phosphorylation and subsequent degradation of its inhibitory subunit (inhibitory protein $\mathrm{kB}, \mathrm{IkB}$ ). IkB is phosphorylated by the correspondent protein serine kinase, acting upstream signaling cascade (IkB kinase $\beta$, IKK- $\beta$ ). $\mathrm{NH}_{2}$-terminal Jun kinases $(\mathrm{JNK}) /$ stress-activated protein kinases (SAPK) belong to a complex superfamily of mitogenactivated serine/threonine protein kinases (MAPK).
This superfamily, including also p38MAPK, consists of stress-activated protein kinases, sensitive to various exogenous and endogenous stress stimuli, including hyperglycemia, ROS, anti-inflammatory cytokines [21].

Activation of signaling cascades including different stress-sensitive serine-threonine kinases, for example, IKK- $\beta$, leads to phosphorylation of numerous cellular targets, including insulin receptor (IR) and proteinssubstrates of insulin receptor (IRS, insulin receptor substrate), IRS-1 and IRS-2 [22]. Phosphorylation of certain serine/threonine residues in IR and IRS molecules dramatically decreases the degree of insulinstimulated phosphorylation of tyrosine residues in these proteins [23]. Thereafter, subsequent association with signaling molecules, situated downstream the signaling cascade, and their activation (first of all, it is phosphoinositide-3-kinase, PI3K) will be perturbed. (PI3K is a lipid kinase, phosphorylating phosphatidylinositol-4,5-phosphate, forming phosphatidylinositol-3,4,5-trisphosphate). As a result, a response to insulin will decrease (insulin resistance) [24]. Thus, antioxidants and pharmacological agents, blocking stress-sensitive kinases, can be considered as potential therapeutic agents preventing the development of insulin resistance. [25]. We have shown earlier in a model of experimental streptozotocin diabetes (diabetes mellitus type 1) in rats that pineal gland hormone melatonin possesses properties of effective direct and indirect antioxidant and can prevent hyperglycemiarelated inhibition of glucose-6-phosphate dehydrogenase and transketolase in liver and regulate nitrogen oxide level [26].

The molecular targets of ROS in the cell are also protein tyrosine phosphatases (PTP), normally acting as negative regulators of insulin catalyzing IR and IRS dephosphorylation.

Peroxisome proliferators-activated receptors (PPAR) play the major role in complex metabolic disorders related to cell resistance to insulin, diabetes mellitus type 2, and metabolic syndrome. PPARreceptors belong to the family of nuclear hormone receptors functioning as ligand-activated transcription factors. The PPAR receptor family, including PPAR$\alpha$, PPAR $-\gamma$, and PPAR- $\delta$, is represented by receptors activated by fatty acids and their metabolites regulating induction of peroxisome enzymes. PPARs play an important role in regulation of activity of genes, thus providing metabolism of glucose, fatty acids, and cholesterol. PPAR $-\alpha$ and PPAR $-\gamma$ are also involved in anti-inflammatory effects. Specific pharmacological correction of PPAR- $\alpha,-\beta,-\gamma$ can be used as a novel promising approach to therapy of diabetes mellitus type 2 and insulin resistance [27]. 


\section{INSULIN-DEPENDENT MECHANISM OF GLUCOSE UPTAKE}

Insulin-dependent signaling cascade. There are two "mirror" signaling cascades underlying homeostasis of glucose metabolism: insulin-mediated glucose uptake (IMGU) in the cells of muscle tissue, liver, and heart and glucose-stimulated insulin secretion (GSIS) in pancreatic $\beta$-cells.

Decrease of cellular response to interaction of insulin with IR (insulin resistance) results in a decrease of the ability of insulin to stimulate glucose uptake and synthesis of glycogen, proteins and lipids [28]. Insulin-dependent signaling cascade regulates glucose level in cells and blood plasma, and its perturbations imminently lead to diabetes mellitus. Metabolic effects of insulin provide homeostasis of glucose metabolism, increasing its consumption in skeletal muscles and suppressing its generation in liver tissue. Insulin resistance reflects a decrease of ability of cells and tissues to respond to physiological level of insulin. Genetic factors, environmental conditions, age and stress contribute to the development of insulin resistance. Perturbations in glucose and lipid metabolism lead to defects in the mechanism of insulin signal transduction and to various pathologies.

Canonical insulin signaling cascade is initiated by interaction of insulin with extracellular $\alpha$-subunit of IR (Fig. 1). This leads to conformational changes in $\beta$ subunit of IR, which possesses its own tyrosine kinase activity and catalyses autophosphorylation of tyrosine residues of IR $\beta$-subunit (NPEY motif). Activated IR phosphorylates proteins of IRS family [29]. IRS phosphorylates specific $\mathrm{SH} 2$-domain of protein tyrosine phosphatase Shp2 and SH3-domain of adaptor protein Grb2. Activated Grb-2 molecule stimulates signaling cascade, linked to small GTP-binding Ras proteins (Ras-GTPases). In this process $\mathrm{SH} 2$ domain of Grb2 protein interacts with adaptor protein Shc and activates Sos, a GTP exchange factor, which binds to Ras-GTPases and activates them, accelerating GDP release. Activation of Ras causes activation of downstream signaling cascade, which stimulates protein kinase Raf and kinase MAPK regulated by extracellular signal (extracellular signal-regulated kinase, ERK). This MAPK-dependent branch of insulin-dependent signaling cascade regulates cellular activities (gene expression, mitosis, cell differentiation and cell survival/apoptosis). Simultaneously, IRS activates PI3K also by interaction with $\mathrm{SH} 2$ protein domain, which is accompanied by an increase of intracellular concentration of phosphatidylinositols (PIP, PIP $_{2}$ and PIP $_{3}$ ) and, in turn, activates PIP-dependent protein kinase 1 (PDK-1). Subsequently, a number of protein serine kinases, including protein kinase $\mathrm{B}(\mathrm{Akt})$ and $\mathrm{PKC}$, are activated. As a result of this reaction sequence, glu- cose transporter GLUT4 is carried from cytoplasm vesicles into cellular plasma membrane (Figs. 1 and 2) [30].

IR activates PI3K isoforms, which are functioning as heterodimers, consisting of catalytic (p110 $\alpha$, $\mathrm{p} 110 \beta, \mathrm{p} 110 \delta$ ) and regulatory (p85 $\alpha, \mathrm{p} 55 \alpha, \mathrm{p} 50 \alpha$, p55 $\gamma$, p $85 \beta$ ) subunits [31]. The signaling pathways listed above, leading to phosphorylation of different cytoplasm proteins and transcription factors, are necessary for realization of metabolic functions by insulin. Insulin signaling cascade is a very complex network of reactions with plenty of feedbacks and interactions with other crucial signaling cascades [32]. It is important that functions of insulin-dependent signaling cascade in heart and skeletal muscles, liver, and vascular endothelial cells are similar, but the biological responses to them are various and tissue-specific (Fig. 2) [30, 33, 34].

Regulation of glycogen synthesis and breakdown. One of the crucial enzymes regulating glucose level in liver cells is phosphorylase (glycogen phosphorylase). Glycogen phosphorylase and glycogen synthase are two main proteins controlling glycogen metabolism (glycogenesis and glycogenolysis). Liver glycogen largely maintains physiological concentrations of glucose. The glycogen molecule consists of glucose residues linked by $\alpha-1,4$-glycoside bonds, specifically split by phosphorylase (the main pathway of glycogen breakdown in the animal cell). Active phosphorylase (phosphorylase $a$ ) is a phosphorylated tetramer. Specific protein phosphatase converts phosphorylase $a$ into inactive phosphorylase $b$ (dimeric molecule), catalyzing hydrolytical removal of phosphate from serine residue. The reverse conversion of inactive phosphorylase $b$ into active phosphorylase $a$ is catalyzed by phosphorylase kinase (phosphorylation of the enzyme). G-6-P and ATP stabilize inactive state of phosphorylase $b$.

Activation of phosphorylase $b$ kinase occurs as a result of its phosphorylation by protein kinase. Protein kinase is activated by cAMP, which induces dissociation of tetrameric protein molecule with release of catalytic subunits. Protein phosphatases, dephosphorylating enzymes, abolish the corresponding effects of protein kinases. Protein phosphatase I (PPI) plays a key role in regulation of glycogen metabolism. (PPI consists of three subunits: catalytic, $R_{G I}$, a subunit with high affinity to glycogen, and inhibitor I - a small regulatory subunit). PPI inactivates phosphorylase kinase and phosphorylase $a$, dephosphorylating these enzymes, thus decreasing the rate of glycogen breakdown. Moreover, PPI dephosphorylates the inactive glycogen synthase $b$, converting it into the active form, thus accelerating glycogen synthesis. PPI is an enzyme regulating the process of glycogen accumulation. The 
insulin-receptor complex;

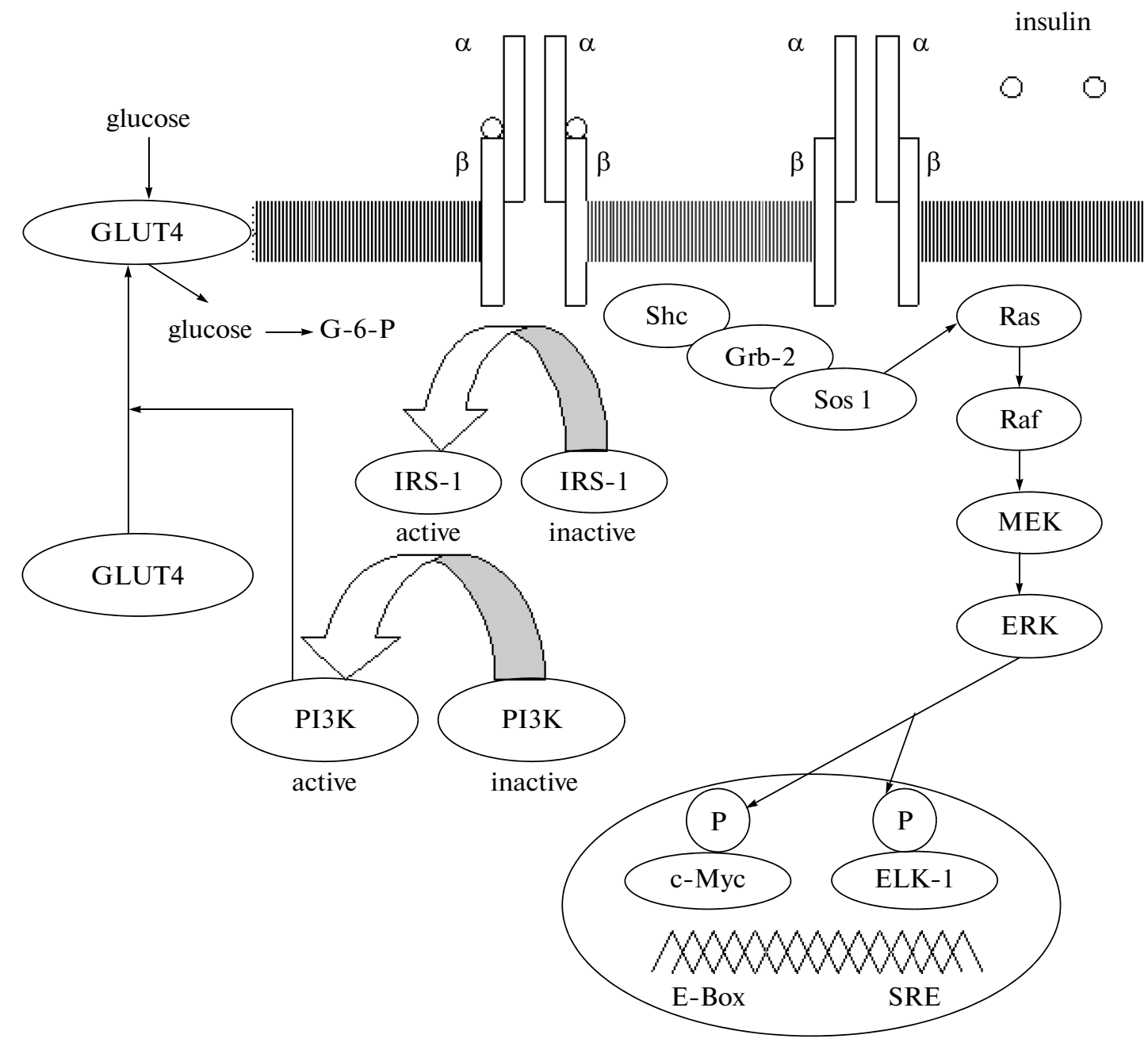

Fig. 1. Insulin signaling cascade includes the following reaction sequence: interaction of insulin with corresponding receptor, leading to phosphorylation of tyrosine of insulin receptor substrates 1 and 2 (IRS-1/2); activation of phosphatydilinositol-3kinase, causing translocation of GLUT4 transporter from intracellular space to plasma membrane of a muscle cell. Binding of insulin with $\alpha$-subunit of IR on the membrane surface leads to autophosphorylation of tyrosine residues of the receptor's $\beta$-subunit possessing its own tyrosine kinase activity and increases tyrosine kinase activity of IR. As a result, IRS-proteins interact with receptors in cytoplasm by their SH2-domains. Subsequent phosphorylation of tyrosine residues of IRS by activated receptor leads to activation of a complex signaling cascade, regulating such important cellular processes, as gene expression, growth and differentiation of pancreatic cells. Proteins of IRS family, IRS-1 and IRS-2, being the most important of them in insulin-sensitive tissues, are docking proteins binding to IR. Anchoring adaptor proteins participating in assembling of protein complexes contain SH2-domain, PH-domain (Pleckstrin homology domain), and PTB-domain recognizing certain amino acid sequences in target proteins. SH2-domains (Src homology domains) recognize and bind phosphorylated tyrosine residues within longer peptide motif of target protein, $\mathrm{PH}$-domains can bind phosphatydilinositol phosphates, $\beta \gamma$-subunits of heterotrimeric G-proteins, PKC. Phosphorylation of numerous tyrosine residues in C-terminal regions of IRS proteins leads to formation of high-specificity binding sites for a number of SH2-domain-containing signaling protein molecules. Phosphatydilinositol-3-kinase, the central insulin-dependent molecule, interacting with IRS and providing metabolic effects of insulin, consists of catalytical and regulatory subunits (p110 and p85, respectively). Activated IRS protein, having also PH-domain, capable of binding products of phosphorylation/dephosphorylation of different centers of inositol ring, interacts with SH2-domain of PI3K catalytical subunit, which is accompanied by increase of activity of p110 - catalytic subunit of the lipid kinase $[55,56]$. Increase of catalytic activity dramatically increases intracellular content of phosphatydilinositol-3,4-disphosphate ( $\left.\mathrm{PIP}_{2}\right)$ and phosphatydilinositol-3,4,5-trisphosphate $\left(\mathrm{PIP}_{3}\right)$. Today there are many identified targets of $\mathrm{PI} 3 \mathrm{~K}$, situated downstream the signaling cascade, for example, such serine/threonine kinases, as phosphoinositide-dependent protein kinase, PKB, PKC, p70S6-kinase, glycogen synthase kinase $[29,30]$. 


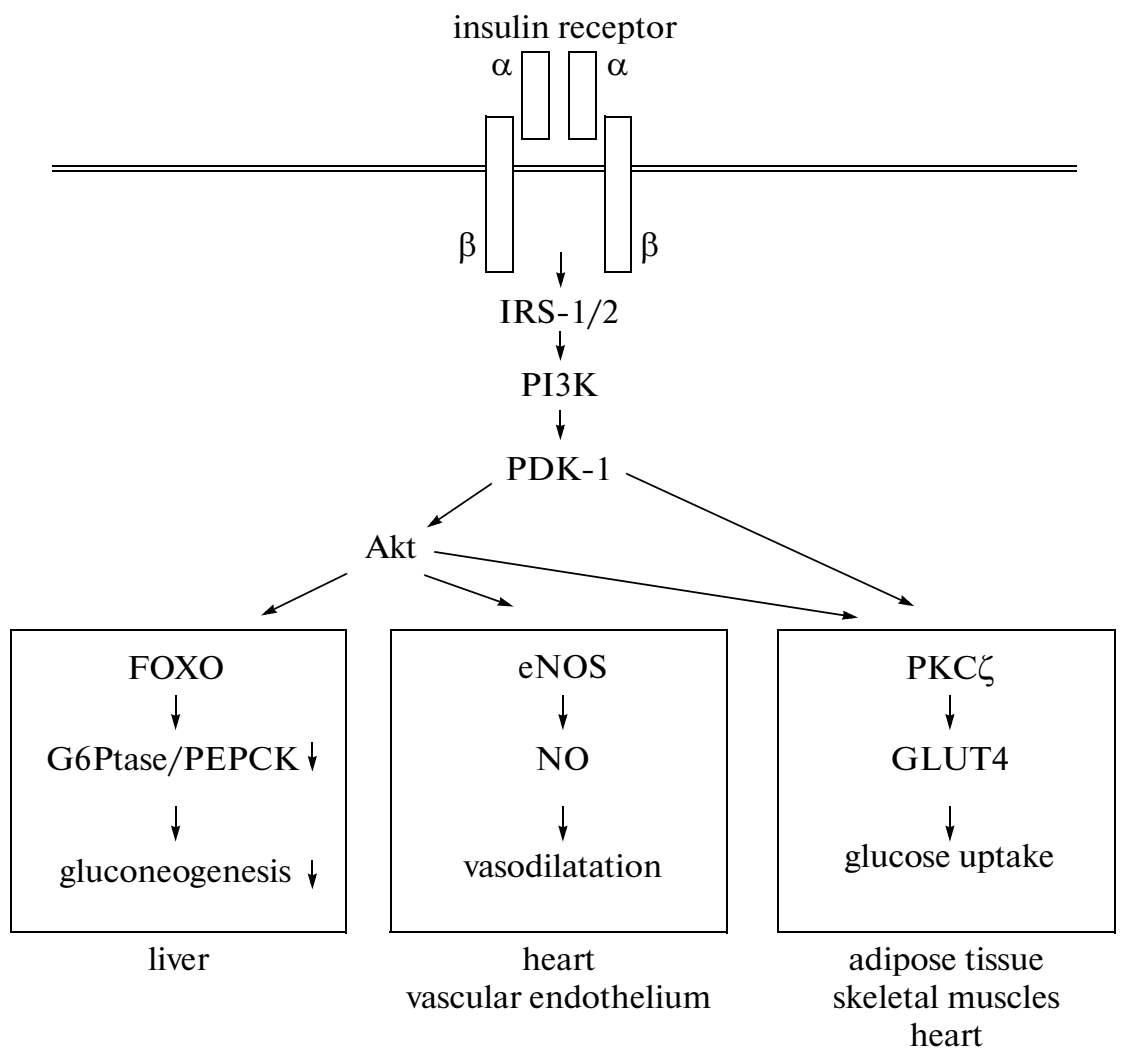

Fig. 2. Insulin-dependent signaling cascade. Metabolic PI3K-dependent branch of the cascade determines tissue-specific effects of insulin: gluconeogenesis in liver, NO production in endothelial and heart cells, glucose uptake by skeletal muscles, adipose tissue, heart tissue [30, 34]. PDK-1, 3-phosphoinositide-dependent protein kinase 1; FOXO, transcription factors belonging to a large family of proteins having conservative DNA-binding domain "fork-head box" (FOX) and regulating many cellular processes. Transcriptional activity of these proteins is regulated by insulin and/or insulin-like growth factor (IGF). PEPCK, phosphoenolpyruvate carboxykinase (the enzyme taking part in gluconeogenesis).

inhibition of PPI is performed by small regulatory subunit - inhibitor I, which suppresses the activity of holoenzyme when being phosphorylated. Phosphorylation of inhibitor I by protein kinase A blocks the catalytic subunits of protein phosphatase I. Protein kinase $\mathrm{A}$ is activated by glucagon or adrenaline by cascade mechanism.

Insulin in turn stimulates glycogen synthesis, activating PPI. Interaction of insulin with the receptor tyrosine kinase - is accomplished by a wave of phosphorylation of different target proteins, activation of insulin-sensitive protein kinase, and phosphorylation of protein phosphatase I in the site, different from the site of phosphorylation by protein kinase A. The result of phosphorylation is the enzyme binding to a glycogen molecule. Dephosphorylation of glycogen synthase, phosphorylase kinase and phosphorylase leads to an increase in glycogen synthesis and the end of its breakdown. The content of active phosphorylase $a$ in liver tissue rapidly decreases after the increase of blood glucose level. Glucose is bound and inhibits glycogen phosphorylase $a$ in liver tissue, shifting the equilibrium from active state to inactive one and facilitating the interaction of the enzyme with protein phosphatase I. The molecular ratio of phosphorylase $a$ /protein phosphatase I is $10 / 1$ [35].

\section{REGULATION OF INSULIN SECRETION BY $\beta$-CELLS. THE ROLE OF NADPH-OXIDASES}

The number of features of glucose metabolism in $\beta$-cells is exclusively important for understanding the role of these cells in maintaining the glucose homeostasis. First of all, such features are: high capacity of the glucose transport system; lack of synthesis of glycogen and fatty acids and of gluconeogenesis; critical role of mitochondrial process, including tricarbonic acid cycle, electron transport, oxidative phosphorylation; $\mathrm{Ca}^{2+}$-independent glucose-induced respiratory burst; voltage-dependent $\mathrm{Ca}^{2+}$ uptake as a signal for insulin secretion; accumulation of malonyl-CoA, acyl-CoA and diacylglycerol as metabolic factors of coupling [36]. Hence, it is clear that complex metabolic reactions in $\beta$-cells, providing energy balance 


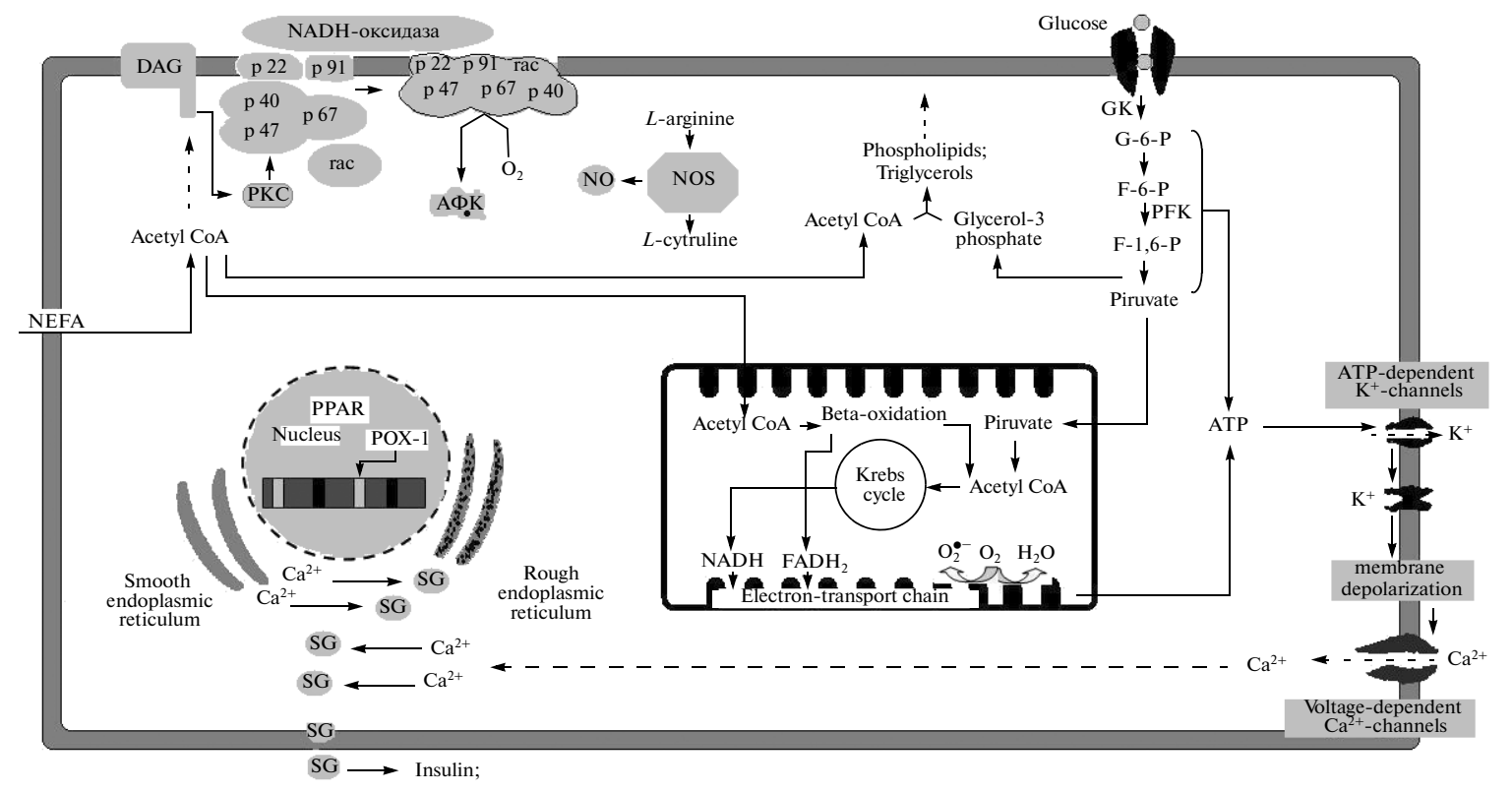

Fig. 3. Glucose-stimulated insulin secretion (GSIS) [37]. Key elements of glucose-stimulated insulin secretion by pancreatic $\beta$-cells: glucose import into the cell by glucose transporter with high $K_{\mathrm{M}}$; glucose phosphorylation by the members of glucokinase (hexokinase) family with high $K_{\mathrm{M}}$; metabolism of formed fructose-6-phosphate in glycolysis and tricarbonic acid cycle; increase of ATP/ADP ratio; closing of ATP-dependent $\mathrm{K}_{+}$channels of the cell membrane; membrane depolarization; opening of voltagedependent $\mathrm{Ca}_{2+}$ channels; the rise in the intracellular $\mathrm{Ca}_{2+}$ concentration causes insulin secretion [34, 37]. GK, glucokinase; G-6-P, glucose-6-phosphate; DAG, diacylglycerol; NEFA, non-esterified fatty acids; PKC, protein kinase C; SG, secretory granules; PPK, phosphofructokinase; F-1,6-P, fructose-1,6-diphosphate; F-6-P, fructose-6-phosphate; NOS, NO-synthase; PPAR, peroxisome proliferator-aNADH-оксидаза->NADH-oxidase;

and functioning of signaling cascades, should be very sensitive to effects of genetic and environmental factors and pharmacological agents.

It is well known that glucose is carried through plasma membranes into insulin-secreting $\beta$-cells of pancreatic Langergans islets by specific transporters, GLUT1 and GLUT2, and is rapidly phosphorylated by glucokinase (hexokinase) having high $K_{\mathrm{m}}$ to glucose (Fig. 3) [37]. (Glucokinase can be considered as a key enzyme for glucose metabolism.) Processes of transport and phosphorylation determine the rate of the flow of glucose, oxidized during glycolysis. Increase of glycolysis rate in $\beta$-cells leads to accumulation of reducing equivalents in cytoplasm and activation of shuttle mechanism of electron transport into mitochondrial matrix, which finally stimulates increase of tricarbonic acid cycle activity and ATP synthesis in mitochondria and also increases the ATP/ADP ratio in cytoplasm. By similar way enhanced oxidation of free fatty acids also leads to an increase of the $\mathrm{NADH} / \mathrm{NAD}$ ratio in mitochondria. The increase of ATP level in cytoplasm is accompanied by closing of ATP-dependent $\mathrm{K}^{+}$channels, which in turn leads to depolarization of plasma membrane, rise in intracellular $\mathrm{Ca}^{2+}$ concentration, activation of protein kinases and insulin exocytosis (Fig. 3) [37, 38]. Fast increase of intracellular $\mathrm{Ca}^{2+}$ concentration via voltage-dependent calcium channels is the basic mechanism of GSIS. However, further increase of intracellular con- centration of $\mathrm{Ca}^{2+}$ can stimulate generation of ROS by mitochondria, because $\mathrm{Ca}^{2+}$ is capable of increasing NADPH-oxidase-dependent generation of ROS by mitochondria, activating PKC, and therefore of increasing the level of oxidative stress and and/or inducing apoptotic cell death.

NADPH-oxidases play a major role in generation of ROS, providing antibacterial effect of specialized phagocytic cells, such as macrophages and neutrophils. "Classic" NADPH-oxidases, traditionally associated with cells of the immune system, are possibly represented by a number of isoforms, among which large families are made out: NOX1, NOX2, and NOX3. Functioning of these isoforms depends on a number of activators. The mentioned enzymatic complexes catalyze the reaction of one-electron oxygen reduction with formation of superoxide anion $\mathrm{O}_{2}^{--}$, using NADPH as electron donor. Integral membrane proteins gp9phox, p22phox, and also p67phox, p47phox, p40 phox, and small GTPases (Rac1 or Rac2) are necessary for regulation of NADPH-oxidase activity. Activation of the enzyme is initiated by phosphorylation of serine or threonine residues of p47phox subunit, catalyzed by PKC, which leads to transport of the enzyme subunits from cytosol to plasma membrane. During activation, six subunits of "classic" NADPH oxidase form an active complex 
producing large amounts of superoxide anion. Regulation of enzyme activity is achieved by two ways:

1) compartmentalization of oxidase subunits with different (membrane and cytosolic) localization,

2) modulation of reversible protein-protein and protein-lipid interactions.

Specific isoforms of $\mathrm{O}_{2}^{\cdot-}$-generating NADPHoxidases (for example, NOX1-3) are the most important source of ROS in non-phagocytic cells, including pancreatic ones. Under the excess of ROS, not only cell damage due to nonspecific oxidation of DNA, proteins, and lipids is observed, but also activation of stress-induced intracellular signaling cascades, such as $\mathrm{NF}-\kappa \mathrm{B}, \mathrm{p} 38 \mathrm{MAPK}, \mathrm{JNK} / \mathrm{SAPK}$, hexosamine, and other signaling pathways. Activation of these cascades leads to increase of expression of many genes, which can be the cause of cell damage and play an important role in the etiology of numerous diseases, particularly in development of long-term diabetes mellitus complications. Results of in vitro and in vivo studies confirm the fact that activation of many stress-dependent signaling pathways leads to the loss of cellular sensitivity to insulin and abnormalities in insulin secretion. Correspondingly, hyperglycemia, free fatty acid level, generation of ROS, oxidative stress, activation of stress-dependent signaling pathways, development of insulin resistance, and dysfunction of $\beta$-cells are thought to correlate with long-term complications of diabetes mellitus is inferred [37, 40].

At the same time, numerous examples prove participation of "non-damaging" physiological level of ROS in cellular signal transduction, regulation of cell growth and programmed cell death, calcium signalization, gene expression [41], and immune responses $[42,43]$.

\section{MOLECULAR MECHANISMS OF INSULIN RESISTANCE}

The resistance of tissues to insulin plays a central role in the development of a number of metabolic disorders and diseases, such as diabetes mellitus type 2, obesity, and metabolic syndrome. The determinative factor in the development of this pathology is an increased level of glucose and free fatty acids in blood plasma and oxidative stress in cells and tissues [44]. Normally, activation of insulin receptors in the cells of insulin-sensitive tissues (muscle tissue, adipose tissue) stimulates glucose oxidation in the glycolytic pathway. However, insulin resistance leads to impairments in insulin-dependent glucose uptake. There are many confirmations for the effect of oxidative stress on intracellular signaling causing the development of inflammatory processes, cell insulin resistance, etc. $[1,45,46]$.

In addition to nonspecific irreversible oxidative damage of DNA, protein, and lipid molecules, reactive oxygen and nitrogen species damage cells and tis- sues indirectly, by activating a number of stress-sensitive cascades. It is supposed that activation of stressdependent signaling cascades during hyperglycemia (and, probably, during increase of free fatty acid contents) plays an essential role in the development of both diabetes type 1 and 2 complications and insulin resistance of tissues in diabetes type 2 [28]. In this case ROS function as signaling molecules.

Defects in different sites of insulin-dependent signaling cascade are considered as a mechanism of the development of insulin resistance [47]. One can draw attention to phosphorylation of serine residues in IRS molecules, IRS degradation, activation of phosphatases (for example, phosphotyrosine phosphatase 1B), suppression of activation of signaling molecules, situated downstream insulin receptors, including Akt and atypical PKC [48, 49].

Phosphorylation of certain serine residues in IRS (by serine/threonine kinases) prevents interaction of IRS with IR, which leads to inhibition of phosphorylation of tyrosine residues in IRS and subsequent suppression of PI3K. Phosphorylation of serine residues in IRS-1/2 proteins leads to insulin resistance due to decrease of insulin-dependent signaling cascade activity, particularly due to inactivation of PI3K, Akt, $\mathrm{PKC}_{\xi}$, and as a result, it decreases cellular glucose uptake, increases glucose production, decreases vasodilatation and insulin secretion [30]. Another more potential mechanism of the development of insulin resistance is activation of pro-inflammatory signaling cascades. For example, Toll-like receptors, playing a key role in initiation of immune response, stimulate pro-inflammatory signaling cascade by subsequent activation of different adaptor protein molecules. The adaptor proteins activate certain kinases, IKK $\beta$ and JNK, which leads to enhancing of signal and direct induction or suppression of certain genes, governing the immune response. As a result of this signaling cascade activation, production of a number of cytokines, for example interleukins, is stimulated [50].

Dysfunction of mitochondria, resulting in an increase of the ROS level, also activates a number of serine protein kinases phosphorylating IRS, which leads to insulin resistance [51]. Moreover, ROS, activating IKK $\beta$, stimulate pro-inflammatory signaling cascade, which phosphorylates serine residues of IRS [52]. Dysfunction of mitochondria promotes accumulation of fatty acid metabolites, DAG and long-chain CoA [53]. DAG, in turn, is an allosteric activator of PKC, also phosphorylating serine residues in IRS-1, which leads to failure of IRS-1 to recruit and activate PI3K and to suppression of insulin signaling [30].

Oxidative stress plays a key role in cell insulin signaling disorders. Activation of JNK signaling cascades inhibits expression of the insulin gene. There are three isoforms of JNK: JNK1, JNK2, JNK3, but only JNK1 is supposed to be involved in the development of diabetes mellitus type 2 [54]. The final result of this mechanism is perturbation in signal transduction for 
translocation of GLUT4 to cellular surface and, thereafter, disturbance in insulin-dependent glucose uptake by the cells $[55,56]$.

Knowledge of the mechanisms of insulin resistance can serve as a basis for the development of pharmacological agents for treatment of this pathology. Usage of antioxidants and pharmacological inhibitors of stressdependent signaling cascades is one of the approaches for the development of such agents. Regulation of functioning and biogenesis of mitochondria can be a novel therapeutic strategy. These new approaches can have an importance for preventing of the development of insulin resistance, $\beta$-cells dysfunction, disturbances of glucose and lipid metabolism in liver, and vasorelaxation [30].

\section{REFERENCES.}

1. Brownlee M. 2005. Banting Lecture 2004. The pathobiology of diabetic complications. A unifying mechanism. Diabetes. 54, 1615-1625.

2. Bojunga J., Dresar-Mayert B., Usadel K.-H., Kusteter K., Zeuzem S. 2004. Antioxidative treatment reverses imbalances of nitric oxide synthase isoform expression and attenuates tissue-cGMP activation in diabetic rats. Biochem. Biophys. Res. Commun. 316, 771-780.

3. Wolff S.P., Dean R.T. 1987.Glucose autoxidation and protein modification: the potential role of "autoxidative glucosylation" in diabetes. Biochem. J. 245, 243250.

4. Wolff, S.P., Jiang Z.Y., Hunt J.V. 1991. Protein glycation and oxidative stress in diabetes mellitus and ageing. Free Radic. Biol. Med. 10, 339-352.

5. Baynes J.W. 2004. The clinical chemome: A tool for the diagnosis and management of chronic disease. Clin. Chem. 50 (7), 1116-1117.

6. Thorpe S.R., Baynes J.M. 2002. Maillard reaction products in tissue proteins: new products and new perspectives. Amino Acids. 25, 275-281.

7. Gerbitz K.D., Gempel K., Brdiczka D. 1996. Mitochondria and diabetes. Genetic, biochemical, and clinical implications of the cellular energy circuit. Diabetes. 45 (2), 113-126.

8. Beyer T.A., Hutson N.J. 1986. Introduction: evidence for the role of the polyol pathway in the pathophysiology of diabetic complications. Metabolism. 35, 1-3.

9. Hamada Y., Nishimura C., Koh N., Sakakibara F., Nakamura J., Tanimoto T., Hotta N. 1998. Influence of interindividual variability of aldose reductase protein content on polyol-pathway metabolites and redox state in erythrocytes in diabetic patients. Diabetes Care. 21, 1014-1018.

10. Chung S.S., Chung S.K. 2003. Genetic analysis of aldose reductase in diabetic complications. Curr. Med. Chem. 10, 1375-1387.

11. Ulrich P., Cerami A. 2001. Protein glycation, diabetes, and aging. Recent Prog. Horm. Res. 56, 1-21.

12. Morgan D., Oliveira-Emilio H.R., Keane D., Hirata A.E., Santos da Rocha M., Bordin S., Curi R., Newsholme P., Carpinelli A.R. 2007. Glucose, palmitate and proinflammatory cytokines modulate production and activity of a phagocyte-like NADPH oxidase in rat pancreatic islets and a clonal $\beta$ cell line. Diabetologia. 50, 359-369.

13. Du X.L., Edelstein D., Rossetti L., Fantus I.G., Goldberg H., Ziyadeh F., Wu J., Brownlee M. 2000. Hyperglycemia-induced mitochondrial superoxide overproduction activates the hexosamine pathway and induces plasminogen activator inhibitor-1 expression by increasing Sp1 glycosylation. Proc. Natl. Acad. Sci. USA. 97, 12222-12226.

14. James L.R., Tang D., Ingram A., Ly H., Thai K., Cai L., Scholey J.W. 2002. Flux through the hexosamine pathway is a determinant of nuclear factor $\kappa \mathrm{B}$-dependent promoter activation. Diabetes. 51, 1146-1156.

15. Goldberg H.J., Whiteside C.I., Fantus I.G. 2002. The hexosamine pathway regulates the plasminogen activator inhibitor- 1 gene promoter and $\mathrm{Sp} 1$ transcriptional activation through protein kinase $\mathrm{C}-\beta$ I and $-\delta$. J. Biol. Chem. 277, 33833-33841.

16. Nisikawa T., Edelstein D., Du X. L., Yamagishi S., Matsumura T., Kaneda Y., Yorek M.A., Beebe D., Oates P.J., Hammes H.P., Giardino I., Brownlee M. 2000. Normalizing mitochondrial superoxide production blocks three pathways of hyperglycaemic damage. Nature. 404, 787-790.

17. Brownlee M. 1995. Advanced protein glycosylation in diabetes and aging. Annu. Rev. Med. 46, 223-234.

18. Stevens M.J., Obrosova I., Feldman E.L., Greene D.A. 2000. The sorbitol-osmotic and sorbitol-redox hypothesis. Diabetes Mellitus: A Fundamental and Clinical Text. Eds LeRoith D., Taylor S.I., Olefsky J.M. Philadelphia: Lippincott Williams \& Wilkins, p. 972-983.

19. Barnes P.J., Karin M. 1997. Nuclear factor kappa B: a pivotal transcription factor in chronic inflammatory diseases. N. Engl. J. Med. 336, 1066-1071.

20. Moxamed A.K., Bierhaus A., Schiekofer S., Tritschler H., Ziegler R., Nawroth P.P. 1999. The role of oxidative stress and NF- $\kappa \mathrm{B}$ activation in late diabetic complications. Biofactors. 10, 157-167.

21. Evans J.L., Goldfine I.D., Maddux B.A., Grodsky G.M. 2003. Are oxidative stress-activated signaling pathways mediators of insulin resistance and $\beta$-cell dysfunction. Diabetes. 52, 1-8.

22. Evans J.L., Maddux B.A., Goldfine I.D. 2005. The molecular basis for oxidative stress-induced insulin resistance. Antioxid. Redox Signal. 7, 1040-1052.

23. Birnbaum M.J. 2001. Turning down insulin signaling. J. Clin. Invest. 108, 655-659.

24. Aguirre V., Uchida T., Yenush L., Davis R., White M. F. 2000. The c-jun $\mathrm{NH}_{2}$-terminal kinase promotes insulin resistance during association with insulin receptor substrate-1 and phosphorylation of Ser307. J. Biol. Chem. 275, 9047-9054.

25. Yuan M., Konstantopoulos N., Lee J., Hansen L., Li Z.W., Karin M., Shoelson S.E. 2001. Reversal of obesity- and diet-induced insulin resistance with salicylates or targeted disruption of IKK $\beta$. Science. 293, 1673-1677.

26. Sudnikovich E.Ju., Maksimchik Yu.Z., Zabrodskaya S.V., Kubyshin V.L., Lapshina E.A., Bryszewska M., Reiter R.J., Zavodnik I.B. 2007. Melatonin attenuates metabolic disorders due to streptozotocin-induced diabetes in rats. Eur. J. Pharmacol. 569, 180-187. 
27. Evans J. L., Lin J.J., Goldfine I. D. 2005. Novel approach to treat insulin resistance, type 2 diabetes, and the metabolic syndrome: simultaneous activation of PPAR $\alpha$, PPAR $\gamma$ and PPAR $\delta$. Curr. Diabetes Rev. 1, 299-307.

28. Evans J.L., Goldfine I.D., Maddux B.A., Grodsky G.M. 2003. Are oxidative stress-activated signaling pathways mediators of insulin resistance and $\beta$-cell dysfunction. Diabetes. 52, 1-8.

29. Bevan P. 2001. Insulin signaling. J. Cell Sci. 114, 14291430.

30. Kim J., Wei Y., Sowers J.R. 2008. Role of mitochondrial dysfunction in insulin resistance. Circ. Res. 102, 401-414.

31. Cantley L.C. 2002. The phosphoinositide 3-kinase pathway. Science. 296, 1655-1657.

32. Sedaghat A.R., Sherman A., Quon M.J. 2002. A mathematical model of metabolic insulin signaling pathways. Am. J. Physiol. Endocrinol. Metab. 283, 10841101.

33. Sowers J.R. 2007. Insulin resistance and hypertension. Am. J. Phiysiol. Heart Circ. Physiol. 293, 2009-2020.

34. McGarry J.D. 2002. Banting Lecture 2001. Dysregulation of fatty acid metabolism in the etiology of type 2 diabetes. Diabetes. 51, 7-18.

35. Chirkin A.A., Danchenko E.A. 2010. Biokhimia: uchebnoe posobie (Biochemistry: A tutorial). M.: Med. Lit., pp. 223-235.

36. Matschinsky F.M. 1996. Banting lecture 1995. A lesson in metabolic regulation inspired by the glucokinase glucose sensor paradigm. Diabetes. 45 (2), 223-241.

37. Newsholme P., Haber E.P., Hirabara S.M., Rebelato E.L.O., ProcopioJ., Morgan D., Oliveira-Emilio H.C., CarpinelliA.R., Curi R. 2007. Diabetes associated cell stress and dysfunction: Role of mitochondrial and non-mitochondrial ROS production and activity. J. Physiol. 583 (1), 9-24.

38. Newsholme P., Brennan L., Bender K. 2006. Amino acid metabolism, $\beta$-cell function, and diabetes. Diabetes. 55 (Suppl. 2), 39-47.

39. Babior B.M. 1999. NADPH oxidase: An update. Blood. 93, 1464-1476.

40. Yoshioka N., Adachi J, Ueno Y., Yoshida K. 2005. Oxysterols increase in diabetic rats. Free Radic. Res. 39, 299-304.

41. Irani K. 2000. Oxidant signaling in vascular cell growth, death, and survival: A review of the roles of reactive oxygen species in smooth muscle and endothelial cell mitogenic and apoptotic signaling. Circ. Res. 87, 179183.

42. Valko M., Leibfritz D., Moncol J., Cronin M.T., Mazur M., Telser J. 2007. Free radicals and antioxidants in normal physiological functions and human disease. Int. J. Biochem. Cell. Biol. 39, 44-84.
43. Schoonbroodt S., Piette J. 2000. Oxidative stress interference with the nuclear factor- $\kappa$ B activation pathways. Biochem. Pharmacol. 60, 1075-1083.

44. Taylor A.J., Ye J.M., Schmitz-Peiffer C. 2006. Inhibition of glycogen synthesis by increased lipid availability is associated with subcellular redistribution of glycogen synthase. J. Endocrinol. 188, 11-23.

45. Fridlyand L.E., Philipson L.H. 2005. Oxidative reactive species in cell injury: Mechanisms in diabetes mellitus and therapeutic approaches. Ann. NY Acad. Sci. 1066, $136-151$.

46. Katakam A.K., Chipitsyna G., Gong Q., Vancha A.R., Gabeta J., Arafat H.A. 2005. Streptozotocin (STZ) mediates acute upregulation of serum and pancreatic osteopontin (OPN): a novel islet-protective effect of OPN through inhibition of STZ-induced nitric oxide production. J. Endocrinol. 187, 237-247.

47. Salniel A.R., Kahn C.R. 2001. Insulin signaling and the regulation of glucose and lipid metabolism. Nature. 414, 799-806.

48. Zhande R., Mitchell J.J., Wu J., Sun X.J. 2002. Molecular mechanism of insulin-induced degradation of insulin receptor substrate 1. Mol. Cell. Biol. 22, 10161026.

49. Vinciguerra M., Foti M. 2006. PTEN and SHIP2 phosphoinositide phosphatases as negative regulators of insulin signaling. Arch. Physiol. Biochem. 112, 89-104.

50. Senn J.J. 2006. Toll-like receptor-2 is essential for the development of palmitate-induced insulin resistance in myotubes. J. Biol. Chem. 281, 26865-26875.

51. Morino K., Petersen K.F., Dufour S, Befroy D., Frattini J., Shatzkes N., Neschen S.,White M.F., Bilz S., Sono S., Pypaert M., Shulman G.I. 2005. Reduced mitochondrial density and increased IRS-1 serine phosphorylation in muscle of type 2 diabetic parents. $J$. Clin. Invest. 115, 3587-3593.

52. Nishikawa T., Araki E. 2007. Impact of mitochondrial ROS production in the pathogenesis of diabetes mellitus and its complications. Antioxid. Redox. Signal. 9, $343-353$.

53. Itani S.I., Ruderman N.B., Schmieder F., Boden G. 2002. Lipid-induced insulin resistance in human muscle is associated with changes in diacylglycerol, protein kinase C, and IкB- $\alpha$. Diabetes. 51, 2005-2011.

54. Hirosumi J., Tuncman G., Chang L., Gorgun C.Z., Uysal K.T., Maeda K., Karin M., Hotamisligil G.S. 2002. A central role for JNK in obesity and insulin resistance. Nature. 420, 333-336.

55. Burks D.J., White M.F. 2001. IRS proteins and $\beta$-cell function. Diabetes. 50 (Suppl. 1), 140-145.

56. Paris M., Bernard-Kargar C., Vilar J., Kassis N., Ktorza A. 2004. Role of glucose in IRS signaling in rat pancreatic islets: specific effects and interplay with insulin. Exp. Diabesity Res. 5, 257-263. 